\title{
Search for MSUSY particles at LEP
}

\section{Barbara Clerbaux*}

CERN-ALEPH, EP division, 1211 Geneva 23, Switzerland

E-mail: 'barbara. clerbaux@cern.ch'

ABSTRACT: Searches for pair production of sleptons, squarks, charginos and neutralinos are performed with the data collected by the ALEPH, DELPHI, L3 and OPAL detectors at LEP at centre-of-mass energies up to $209.2 \mathrm{GeV}$. No evidence for any such signal is observed. Preliminary LEP2 mass lower limits are reported in the framework of the minimal supersymmetric model.

\section{Introduction}

During the year 2000, its last year of running, the LEP collider operated at its maximum energy in the electron-positron centre-of-mass (up to $209.2 \mathrm{GeV}$ ). The data collected by the 4 experiments (ALEPH, DELPHI, L3 and OPAL) are divided into 3 energy samples: 205, 207 and $208 \mathrm{GeV}$, corresponding respectively to an integrated luminosity of 75,120 and 9 $\mathrm{pb}^{-1}$ per experiment. These high energy data provide an opportunity to extend the region to search for new particles and in particular the ones predicted by the supersymmetric theories (SUSY), such as the minimal supersymmetric extension of the standard model (MSSM) [1].

In the MSSM, each standard model (SM) fermion chirality state has a scalar supersymmetric partner (sfermion right and left, $\tilde{f}_{R}$ and $\tilde{f}_{L}$ ), which are weak eigenstates and can mix to form mass eigenstates. The mixing is expected to be relevant for the scalar top (stop, $\tilde{t}$ ), scalar bottom (sbottom, $\tilde{b}$ ) and scalar tau (stau, $\tilde{\tau}$ ). The charginos, $\tilde{\chi}_{j}^{ \pm}$, are mass eigenstates formed by mixing of the field of the fermionic partners of the $\mathrm{W}$ boson and those of the charged Higgs boson. Fermionic partners of the photon, $\mathrm{Z}$ and neutral Higgs bosons mix to form mass eigenstates called neutralinos, $\tilde{\chi}_{i}^{0}$. The indexes $i$ and $j$ are ordered by increasing mass. The R-parity is assumed to be conserved, which implies that the supersymmetric particles are pair produced and the lightest supersymmetric particle (LSP) is stable. The LSP is considered to be the lightest neutralino or the scalar neutrino (sneutrino, $\tilde{\nu}$ ) if it is sufficiently light.

${ }^{*}$ Speaker. 
Motivated by Grand Unification and to simplify the physics interpretation, the constrained MSSM (CMSSM) assumes unification of masses and couplings at the GUT scale. The number of SUSY parameters reduces then to 6, which are $\mathrm{SU}(2)$ gaugino mass parameter at $\mathrm{EW}$-scale $\mathrm{M}_{2}$, common scalar mass at GUT scale $\mathrm{m}_{0}$, common trilinear coupling $\mathrm{A}_{0}$, mass of the $\mathrm{CP}$ odd Higgs boson $\mathrm{m}_{A}$, and the parameters $\mu$ and $\tan \beta$ representing respectively the mixing parameter of the 2 Higgs doublet fields and the ratio of the VEV's of the 2 Higgs doublets.

Direct searches for sleptons, squarks, charginos and neutralinos performed at LEP are reported. In order to increase the sensitivity for the searches, most of the single experiment results are combined (labelled in the following "ADLO") by the means of the Likelihood Ratio method [2].

\section{Search for sfermions}

In this section, all SUSY particles (except the LSP) are supposed to be heavier than the considered sfermion. Sfermions are pair produced at LEP, $\mathrm{e}^{+} \mathrm{e}^{-} \rightarrow \tilde{f} \tilde{\bar{f}}$, via s-channel exchange of a virtual photon or $\mathrm{Z}$, whereas the t-channel exchange of a neutralino contributes (with a constructive interference) in case of selectron ( $\tilde{e})$ production, making possible, for this flavour, a mixed production $\tilde{e}_{R} \tilde{e}_{L}$. All sfermions, but the stop, decay dominantly as $\tilde{f} \rightarrow f \tilde{\chi}^{0}$. The final state is then characterised by missing energy (carried by the $\tilde{\chi}^{0}$ ) and the event properties depend significantly on $\Delta M$, the mass difference between the sfermion and the LSP. When $\Delta M$ is large, a substantial amount of energy is visible in the detector and the main backgrounds from $\mathrm{SM}$ processes are $\mathrm{WW}, \mathrm{We} \nu, \mathrm{Z} \gamma^{*}$ and $\mathrm{q} \overline{\mathrm{q}}(\gamma)$ events. At small $\Delta M$, the energy available in the visible system is small and the signal events are similar to the $\gamma \gamma$ events.

\subsection{Sleptons}

Slepton $(\tilde{\ell})$ pair production leads to a final state of 2 acoplanar leptons of the same flavour and missing energy. No signal for these processes is found and a $95 \%$ CL upper limit on the cross section of $0.02(0.1) \mathrm{pb}$ is obtained in case of selectron and smuon (stau) productions, in most of the mass plane $\left(m_{\tilde{\ell}}, m_{\tilde{\chi}^{0}}\right)$ (with $\Delta M>10 \mathrm{GeV} / \mathrm{c}^{2}$ ). Mass lower limits are derived in the MSSM for $\mu=-200 \mathrm{GeV} / \mathrm{c}^{2}$ and $\tan \beta=1.5$, assuming no mixing (see Fig. $i_{-1}^{1}($ left $)$ ). For $\Delta M>10 \mathrm{GeV} / \mathrm{c}^{2}$ the preliminary observed (expected) ADLO combined limits are [2]: $m_{\tilde{e}}>99(99) \mathrm{GeV} / \mathrm{c}^{2}, m_{\tilde{\mu}}>95(91) \mathrm{GeV} / \mathrm{c}^{2}, m_{\tilde{\tau}}>80(85) \mathrm{GeV} / \mathrm{c}^{2}$.

Conservatively the limits are given for right-handed slepton production (characterised by a lower cross section compared to left-handed production). In case of stau mixing, such that the cross section is minimum, the limit given above typically degrades by $2 \mathrm{GeV}$.

\subsection{Squarks}

If the stop is lighter than all other SUSY particles (except the LSP), it decays only via loop diagrams, $\tilde{t} \rightarrow \mathrm{c} \tilde{\chi}^{0}$, or via virtual chargino exchange, $\tilde{t} \rightarrow \mathrm{b} \ell \tilde{\nu}$, when kinematically possible. Events with acoplanar jets and acoplanar jets plus 2 leptons are then signatures 

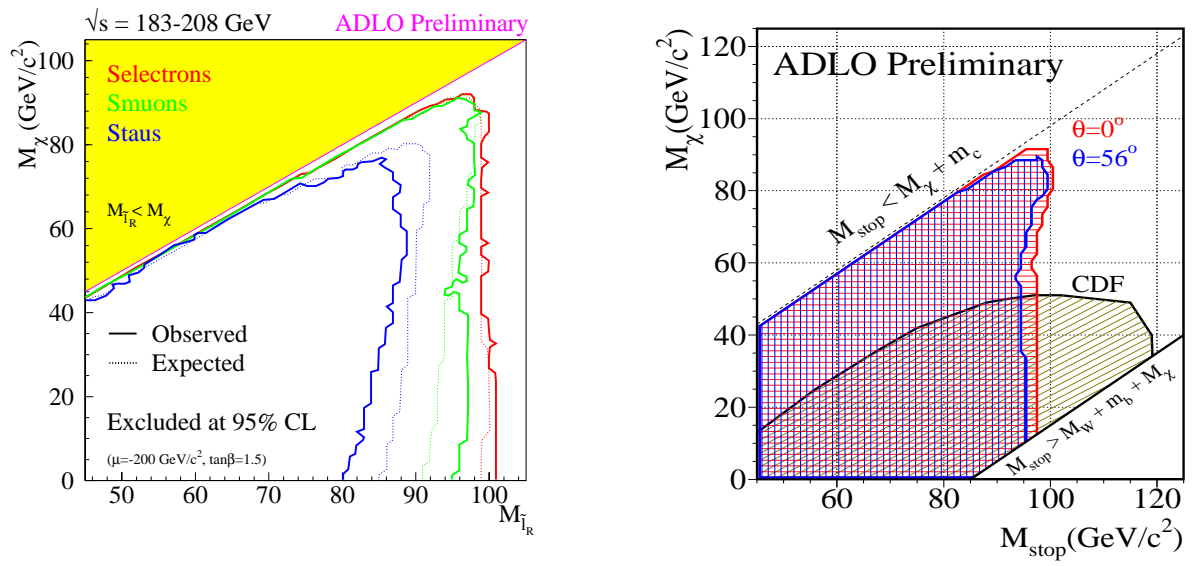

Figure 1: Left: LEP $95 \%$ CL lower limit on the right-handed $\tilde{e}, \tilde{\mu}$ and $\tilde{\tau}$ masses (from the right to the left) in the plane $\left(\mathrm{m}_{\tilde{\chi}^{0}}, \mathrm{~m}_{\tilde{\ell}}\right)$. Selectron cross section and $\operatorname{BR}\left(\tilde{\ell} \rightarrow \ell \tilde{\chi}^{0}\right)$ are computed for $\mu=-200 \mathrm{GeV} / \mathrm{c}^{2}$ and $\tan \beta=1.5$. Right: LEP $95 \% \mathrm{CL}$ lower limits on the stop mass in the plane $\left(\mathrm{m}_{\tilde{\chi}^{0}}, \mathrm{~m}_{\tilde{t}}\right)$ in case of no mixing $(\theta=0)$ and for a mixing such that the cross section is minimum $\left(\theta=56^{\circ}\right)$. The Tevatron result $[3]$ is also shown.

for squark production. For $\tilde{b} \rightarrow \mathrm{b} \tilde{\chi}^{0}$ searches, the analysis uses the lifetime content in the b-jet.

The non-observation of any excess compared to the SM background is translated into a lower limit on the squark mass (see for example Fig. c $\tilde{\chi}^{0}$ ). For $\Delta M>10 \mathrm{GeV} / \mathrm{c}^{2}$ the preliminary observed ADLO combined limits in case of no mixing (for mixing such that the cross section is minimal) are [2]: $m_{\tilde{t}}>97(95) \mathrm{GeV} / \mathrm{c}^{2}$ for $\tilde{t} \rightarrow \mathrm{c} \tilde{\chi}^{0}, m_{\tilde{t}}>97(95) \mathrm{GeV} / \mathrm{c}^{2}$ for $\tilde{t} \rightarrow \mathrm{b} \ell \tilde{\nu}, m_{\tilde{b}}>100(92) \mathrm{GeV} / \mathrm{c}^{2}$ for $\tilde{b} \rightarrow \mathrm{b} \tilde{\chi}^{0}$.

The 4 body decay of the stop, $\tilde{t} \rightarrow \mathrm{b} \tilde{\chi}^{0} f \bar{f}^{\prime}$ dominates over the $\tilde{t} \rightarrow \mathrm{c} \tilde{\chi}^{0}$ mode in a large area of MSUSY parameter space, if the exchanged particles $\left(t, \tilde{\chi}^{+}, \tilde{b}, \tilde{\ell}\right)$ are not much heavier than the stop (see recent calculations in [4]). A new analysis is performed by the ALEPH Collaboration, searching in the data collected in the year 2000 for topologies with multijets and missing energy. Two cases are considered for the decay mode of the exchanged chargino (dominant diagram): using $\mathrm{W}^{*} \mathrm{BR}$ or $100 \%$ leptonic BR. No signal is observed, and preliminary lower limits on the stop mass are extracted: 80 and $86 \mathrm{GeV} / \mathrm{c}^{2}$ for the $\mathrm{W}^{*}$ and leptonic $\mathrm{BR}$ respectively (in case of mixing, the limits degrade to 72 and $\left.77 \mathrm{GeV} / \mathrm{c}^{2}\right)[5]$.

In the squark searches, LEP extends the excluded region by Tevatron at small mass difference $\left(\Delta M<40 \mathrm{GeV} / \mathrm{c}^{2}\right)[2]$, as shown in Fig. $\stackrel{\overline{2}}{\mathrm{z}}$ (left).

\section{Search for neutralinos and charginos}

Charginos and neutralinos are pair produced at LEP via the s-channel exchange of $\mathrm{Z}^{*}$ (or a $\gamma^{*}$ in case of the chargino) and via the t-channel exchange of a $\tilde{\nu}$ (for the charginos) or a $\tilde{e}$ (for the neutralinos). The chargino cross section is large except if the $\tilde{\nu}$ is light, in which 
case the cross section is reduced because of the destructive interference between the $\mathrm{s}$ and t-channels.

The chargino $\tilde{\chi}_{1}^{+}$decays into $\tilde{\chi}_{1}^{0} \ell^{+} \nu$, or $\tilde{\chi}_{1}^{0} \mathrm{q} \bar{q}^{\prime}$, via a $\mathrm{W}^{*}$ boson, a slepton $(\tilde{\ell}, \tilde{\nu})$ or a squark. The neutralino $\tilde{\chi}_{2}^{0}$ decays into $\tilde{\chi}_{1}^{0} \nu \bar{\nu}, \tilde{\chi}_{1}^{0} \ell \ell$ or $\tilde{\chi}_{1}^{0} q \bar{q}$ through a $Z^{*}$ boson, a sneutrino, a slepton, a squark or a neutral SUSY Higgs boson $\left(\mathrm{h}^{0}\right.$ or $\left.\mathrm{A}^{0}\right)$. In much of the MSSM parameter space, $\tilde{\chi}_{1}^{+}$decays via a $\mathrm{W}^{*}$ boson and $\tilde{\chi}_{2}^{0}$ decays via $\mathrm{Z}^{*}$ are dominant. For small slepton masses, decays into leptons become important. If the sneutrino is lighter than the chargino, the 2-body decay $\tilde{\chi}_{1}^{+} \rightarrow \tilde{\nu} \ell^{+}$dominates. The chargino pair production is then characterised by a final state with 4 jets, 2 jets plus a lepton or 2 leptons, and missing energy. In case of neutralino $\left(\tilde{\chi}_{2}^{0} \tilde{\chi}_{1}^{0}\right)$ production, the topologies are 2 jets or 2 leptons, and missing energy. The standard selections are categorised by event topologies and mass differences $\left(\Delta M_{+}=\mathrm{m}_{\tilde{\chi}_{1}^{+}}-\mathrm{m}_{\tilde{\chi}_{1}^{0}}\right.$ and $\left.\Delta M_{0}=\mathrm{m}_{\tilde{\chi}_{2}^{0}}-\mathrm{m}_{\tilde{\chi}_{1}^{0}}\right)$.

The contribution from background processes depends on the $\Delta M$ region. In the small $\Delta M$ region, $\gamma \gamma$ processes are dominant. To recover sensitivity in this region, dedicated analyses of chargino mass-degenerated with the $\tilde{\chi}_{1}^{0}$ are introduced, which reduce the contribution from $\gamma \gamma$ background by tagging an energetic photon from initial state radiation. At very low $\Delta M_{+}\left(\Delta M_{+}<\mathrm{m}_{\pi}\right)$, the chargino can have a sizable lifetime and searches for heavy long living charged particles are performed.

No signal for chargino and neutralino productions is observed at LEP, and preliminary $95 \%$ CL upper limits on the cross sections are extracted, by example by the OPAL experiment, to be around $0.2 \mathrm{pb}$ at high $\Delta M$ (see Fig. 12:(right)), and, in case of charginos, less than few $\mathrm{pb}$ for $\Delta M_{+}<5 \mathrm{GeV} / \mathrm{c}^{2}$ [7]. The limits are obtained assuming that the charginos and neutralino decay via a $\mathrm{W}^{*}$ and a $\mathrm{Z}^{*}$ bosons respectively.

As the chargino and neutralino cross sections and decays depend on the MSSM parameters, a scan on theses different parameters has to be performed in order to extract mass limits. This analysis is reported in the next article.
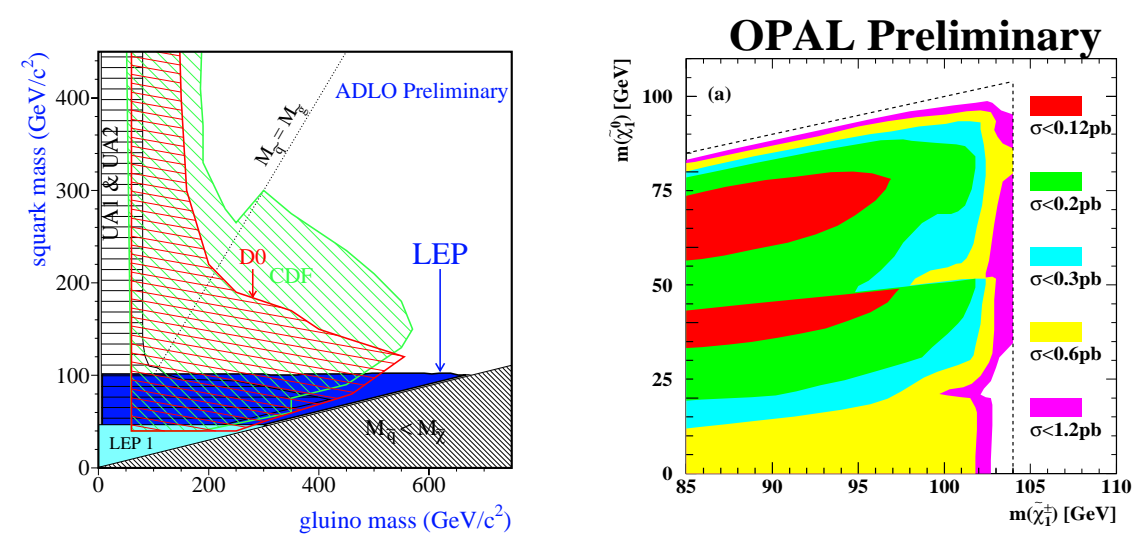

Figure 2: Left: Region excluded at $95 \%$ CL by LEP and Tevatron [6] experiments in the plane of the degenerate squark mass and the gluino mass. Right: $95 \%$ CL upper limit on the chargino cross section in the plane $\left(\mathrm{m}_{\tilde{\chi}^{0}}, \mathrm{~m}_{\tilde{\chi}^{+}}\right)$assuming that charginos decay via a $\mathrm{W}^{*}$ bosons. 


\section{Absolute mass limits}

In the framework of the MSSM, an absolute lower limit on the stop mass and on selectron and sneutrino masses are extracted from the data after a scan on the relevant parameter space. The results of the standard searches described in the previous sections are used. New dedicated analyses are performed in order to exclude some regions in the parameter space, in particular for sfermions at low $\Delta M$ and in case of cascade decay of the selectron.

\subsection{Stop limit}

At very small $\Delta M$, the decay $\tilde{t} \rightarrow \mathrm{c} / \mathrm{u} \tilde{\chi}^{0}$ is relevant and the stop has a sizable lifetime. Searches for stable and metastable hadrons are performed by ALEPH. The intermediate lifetime selection addresses the case of stop hadrons decaying within the tracker volume, at a significant distance from the interaction vertex. The analysis selects tracks with large impact parameter, originating from the stop decay. When the stop decay length is larger than the detector size, the charged stop-hadron behaves like a heavy stable charged particle, and is identified by a high specific ionisation in the tracker (Time Projection Chamber). In all cases the number of candidates observed is in agreement with the background expected from the SM. In the framework of the MSSM, a preliminary absolute lower limit on the stop mass is found to be $65 \mathrm{GeV} / \mathrm{c}^{2}$, valid for all values of $\Delta M, \mu$ and $\tan \beta[5]$.

\subsection{Selectron and sneutrino limits}

The absolute mass lower limit on selectron and sneutrino is obtained in the framework of CMSSM, where $\tilde{e}_{R}, \tilde{e}_{L}$ and $\tilde{\nu}$ masses depend on $\mathrm{m}_{0}, \mathrm{M}_{2}$ and $\tan \beta$ parameters.

To recover sensitivity at small $\Delta M\left(\Delta M<5 \mathrm{GeV} / \mathrm{c}^{2}\right)$, a dedicated search for associate production of $\tilde{e}_{R} \tilde{e}_{L}$ is performed. The mass of the $\tilde{e}_{L}$ being higher than the one of the $\tilde{e}_{R}$, this leads to a final state of one visible electron in the detector coming from the decay of the $\tilde{e}_{L}$ (the $\tilde{e}_{R}$ decay electron being soft and invisible). New analyses are developped to exclude some regions in the parameter space, mainly where selectrons decay in cascades and the chargino is mass degenerated with the sneutrino. The sensitivity is recovered by searching for $\tilde{\chi}_{3}^{0} \tilde{\chi}_{1}^{0}$ production, with leptonic cascade decay of the $\tilde{\chi}_{3}^{0}$. Depending on $\Delta M$, topologies of the final state are then 1 to 4 visible leptons, or 1 to 2 visible leptons and a photon. No signal is found in all these topologies.

The absolute lower limit on the selectron mass is presented in Fig. 1 (1) (left) as a function of $\tan \beta$. At low $\tan \beta$, the limit is given by the $\tilde{e}_{R} \tilde{e}_{L}$ analysis and at high $\tan \beta$, by the standard search for $\tilde{e}_{R} \tilde{e}_{R}$. The effect of the Higgs constraints is also shown for 2 different top masses: it excludes the low $\tan \beta$ region. The preliminary ALEPH absolute lower limit for the selectron mass is $75(77) \mathrm{GeV} / \mathrm{c}^{2}$ for $\mathrm{m}_{\text {top }}=175(180) \mathrm{GeV} / \mathrm{c}^{2}$ [8]. An ALEPH absolute mass lower limit for the sneutrino is extracted by the same procedure and is found to be $83 \mathrm{GeV} / \mathrm{c}^{2}$ (the limit is found at high $\tan \beta$ ) [8].

The DELPHI Collaboration performed the same analysis with the same hypothesis (CMSSM) but restricted themself at high $\Delta M: \mathrm{m}_{\tilde{e}_{R}}-\mathrm{m}_{\tilde{\chi}^{0}}>10 \mathrm{GeV} / \mathrm{c}^{2}$. In that case, the preliminary DELPHI absolute mass lower limits for the selectron and the sneutrino are 

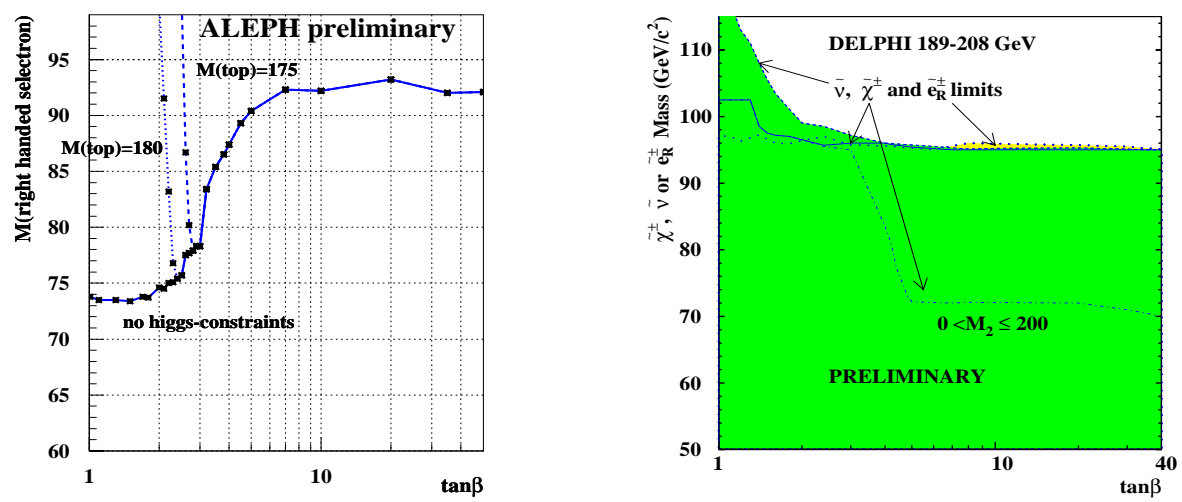

Figure 3: Left: $95 \%$ CL absolute lower limit on the selectron mass as a function of $\tan \beta$, with and without the Higgs constraints (for $\mathrm{m}_{\text {top }}=175$ or $180 \mathrm{GeV} / \mathrm{c}^{2}$ ), the limit is valid for all $\Delta M$ $=\mathrm{m}_{\tilde{e}_{R}}-\mathrm{m}_{\tilde{\chi}^{0}}$. Right: $95 \% \mathrm{CL}$ absolute lower limit on the sneutrino mass as a function of $\tan \beta$ (dark shading and the dashed curve), together with limit on the right-handed slepton mass (dotted curve and light shading), the limits are valid for $\Delta M=\mathrm{m}_{\tilde{e}_{R}}-\mathrm{m}_{\tilde{\chi}^{0}}>10 \mathrm{GeV} / \mathrm{c}^{2}$.

both $95 \mathrm{GeV} / \mathrm{c}^{2}[9]$. The results of the scan is presented in Fig. is. (right) as a function of $\tan \beta$.

\section{Conclusions}

Searches for pair production of sleptons, squarks, charginos and neutralinos are performed with the data collected by the ALEPH, DELPHI, L3 and OPAL detectors at LEP at centreof-mass energies up to $209.2 \mathrm{GeV}$. No signal is observed. The preliminary LEP2 combined limits are given, which improve significantly on the constraints set previously.

\section{References}

[1] Supersymmetry and supergravity, Ed. M. Jacob, North-Holland and World Scientific, 1986.

[2] LEP SUSY Working Group WEB page: http://lepsusy.web.cern.ch/lepsusy/

[3] CDF Coll., Phys. Rev. Lett. 84 (1999) 5704.

[4] C. Boehm, A. Djouadi and Y. Mambrini, Phys. Rev. D61 (2000) 095006.

[5] ALEPH 2001-065, CONF. 045.

[6] CDF Coll., FERMILAB-PUB-01/084-E, Submitted to Phys. Rev. Lett., D0 Coll., Phys. Rev. Lett. 75 (1995) 618, D0 Coll., Phys. Rev. Lett. 83 (1999) 4937.

[7] OPAL PN470, CONF. 027.

[8] ALEPH 2001-067, CONF. 047.

[9] DELPHI 2001-085, CONF. 513. 